\title{
Instituciones. Reglas y procesos
}

\author{
Institutions. Rules and processes
}

Ma Teresa López de la Vieja de la Torre

Universidad de Salamanca tlv@usal.es

DOI: http://doi.org/10.15366/bp2019.20.003

Bajo Palabra. II Época. No20. Pgs: 51-64 


\section{Resumen}

Lo institucional es una manifestación del poder. La tesis de $\mathrm{H}$. Arendt a este respecto tenía una segunda parte: las instituciones decaen e incluso se petrifican en cuanto el poder vivo, del pueblo, deja de sostenerlas. La reciente situación de crisis, en la esfera política nacional e internacional, puede tener su origen en la pérdida de apoyo al poder que se materializa en sistemas institucionales. Sin embargo, su erosión dependerá de varios factores, no sólo del "poder vivo", como lo denominaba H. Arendt. Esos procesos de desintitucionalización son complejos $\mathrm{y}$, por ello, el análisis tendría que considerar: (1) el papel integrador de las instituciones, ya que articulan las expectativas reciprocas de los agentes, (2) las instituciones como sistemas de reglas que definen derechos y obligaciones, (3) el papel de las "reglas constitutivas". De manera general, conviene tener en cuenta la normatividad de estos sistemas públicos de reglas, con poder deóntico.

Palabras Clave: Institucionalización, desinstitucionalización, sistemas de reglas, normatividad, legitimidad

\section{Abstract}

Institutions are a manifestation of power. According to $\mathrm{H}$. Arendt, a second aspect has to be considered; institutions could decay and even petrify when the living power, the people's power, doesn't uphold them. The recent crisis in the national and the international political sphere, could derive from insufficient backing of power in institutional systems. However, their erosion would depend on several factors, not only of the "living power", as H. Arendt stated. Deinstitutionalization processes are complex phenomena and, consequently, they require several levels of analysis: (1) institutions perform integration functions, articulating reciprocal expectations of agents, (2) as systems of rules, institutions define rights and duties (3) the role of "constitutive rules". In general, the normativity or deontic power of public systems of rules has to be taken into account.

Keywords: Institutionalization, deinstitutionalization, system of rules, normativity, legitimation 


\section{Introducción}

"El apoyo del pueblo presta poder a las instituciones de un país. Este apoyo es la continuación del consenso que ha creado las leyes. ...Todas las instituciones políticas son manifestaciones y materialización de poder; quedan petrificadas y decaen en cuanto el poder vivo del pueblo deja de sostenerlas." ${ }^{1}$

Lo institucional es unA MANifestación más del poder. La tesis de H. Arendt tenía una segunda parte: las instituciones decaen y se petrifican en cuanto el poder vivo, el del pueblo, deja de sostenerlas. Este poder otorgado sería, pues, la clave de la dinámica en la esfera pública. La reciente situación de crisis, fuera y dentro de la esfera política, vendría a confirmar la opinión de $\mathrm{H}$. Arendt sobre las bases del poder institucional. La pérdida de apoyo o de consenso en torno a las instituciones muestra, además, el declive de la estructura y de las funciones a realizar por los partidos y los sistemas políticos tradicionales. Ahora bien, su erosión o deterioro dependerá de varios factores, no sólo del desestimiento del "poder vivo", en los términos de H. Arendt.

Esos procesos - pues de procesos se trata- son complejos. Lo son también aquellos que llevan a la desintitucionalización; en consecuencia, el análisis teórico tendría que considerar cuáles son las funciones de los sistemas, desde la capacidad de integración hasta la normatividad y su potencial constructivo: (1) El papel integrador de las instituciones articula las expectativas recíprocas de los agentes, tanto en el ámbito social como en el político. (2) Las instituciones son sistemas de reglas, capaces de definir los derechos y las obligaciones de quienes se encuentren dentro del sistema, (3) sin olvidar el papel de las "reglas constitutivas". Esto es, aquellas que generan nuevas formas de comportamiento. Por todo ello, conviene tener en cuenta la normatividad o "poder deóntico" de los sistemas públicos de reglas.

\section{Instituciones}

EN DETERMINADAS CIRCUNSTANCIAS, procesos en marcha acabarán debilitando y restando fuerza a las instituciones. Dependerá bastante de cuál sea el momento y el

${ }^{1}$ Arendt, H.: On Violence, Orlando, Harcourt Brace Jovanovich Publishers, 1969, p. 41 
lugar en los que esto se produzca. Así ha sucedido al menos en tres casos, los movimientos de protesta del año 2011, las críticas reiteradas al dominio patriarcal y el reciente deterioro de las políticas de la Unión Europea. En los tres, las tensiones han sido, son perceptibles. ¿Hay forma de evitarlas? En principio, las instituciones están siempre sujetas a una doble dinámica, de consolidación y de cambio. Solo que los procesos avanzan, paso a paso, hacia la estabilidad o hacia la erosión de las instituciones; por eso, por su carácter gradual, pueden resultar solo en parte visibles.

Al final ¿pesarán más los procesos de adaptación o la ruptura de los sistemas? Las instituciones han de adaptarse a entornos cambiantes y, al mismo tiempo, tienden a mantener su autonomía ${ }^{2}$. Si esto es así ¿cómo hacer compatibles la estabilidad y el cambio? En general, los procesos de institucionalización $-\mathrm{y}$ los que van en sentido opuesto- surgen en un entorno que tiende a ser estable y, a la vez, alimenta tensiones. En cualquier caso, las instituciones mantienen rutinas y expectativas reciprocas, pues estos procesos de "habituación" cumplen varias funciones ${ }^{3}$. ¿Qué tipo de funciones?

Para empezar, las instituciones suelen tipificar las conductas, aportando estabilidad y, en fin, un marco que sea suficientemente claro para la interacción entre los agentes. De este modo, contribuyen a reducir, en parte, la incertidumbre que acompaña a la toma de decisiones y a la acción. Es importante, pues el orden social y el político dependen casi siempre de ese marco institucional, dentro del cual se definen los papeles, también los derechos y deberes de cada uno de los agentes.

Es más, esa forma de definir las expectativas reciprocas se convierte en un mecanismo de integración. ¿Cómo funciona? Gracias al conjunto de papeles y reglas, se crea una situación compartida por y para una pluralidad de agentes ${ }^{4}$. En medio de la diversidad, es posible contar con algo en común. Pero la existencia de un sistema estable para quienes tienen intereses diferentes no evita que, llegadas las circunstancias, los procesos de institucionalización sean reversibles. Lo son, al igual que otros procesos. De ahí surge la otra tendencia, la desinstitucionalización. Por lo tanto, la habituación y, luego, las estructuras que regulan los intercambios suelen estar sujetas a presiones de todo tipo, internas y externas.

Según esto, los marcos estables pueden dejar de serlo en algún momento. El problema es predecir con exactitud cuándo llegará. Tampoco está claro cómo y hasta

\footnotetext{
${ }^{2}$ Huntington, S.: Political Order in Changing Societies, New Haven, Yale University Press, 1968, pp. 1-92

3 Berger, P., Luckmann, Th.: The Social Construction of Reality, London, Penguin, 1991, p. 70

4 Parsons, T.: On Institutions and Social Evolution, Chicago. The University of Chicago Press, 1982, pp. 117-128
} 
qué punto afectará a las instituciones: entre la estabilidad y el cambio, caben varias posibilidades. Afectarán de manera distinta a cada una de las funciones que desempeña cada institución, dependiendo de cuál sea el grado de desviación con respecto a los patrones "habituales". La conclusión es que habrá que prestar atención a todos los procesos, aquellos que crean los marcos o las estructuras y a los otros, los que llevan a su declive. Algunos ejemplos permitirán ver más de cerca la dinámica de institucionalización/desintitucionalización.

\subsection{Procesos}

EN EL AÑo 2OI I, las protestas y movimientos sociales surgidos tanto en el Cairo, en la plaza Tahrir, luego en Madrid (el movimiento 15-M), en Atenas (la plaza Syntagma) y en Nueva York (Occupy Wall Street), reaccionaron casi al mismo tiempo. Lo hicieron, sin embargo, de forma distinta. Las protestas mostraron hasta dónde había llegado la erosión de los marcos institucionales, con fracturas económicas, sociales, culturales y políticas. Algunos conflictos no eran del todo nuevos, lo eran los factores desencadenantes, en especial las políticas de austeridad. También eran distintas algunas de las consecuencias -entre otras, una clase social víctima de la precariedad ${ }^{5}-\mathrm{o}$ las formas de movilización. En el punto de mira estaban las instituciones y todo un sistema. Ya lo habían estado en el discurso que había llamado a reaccionar de forma decidida contra los excesos del mercado y en defensa de los derechos fundamentales. jIndignaos! ${ }^{6}$

En la esfera pública, el impacto de estos movimientos ha sido desigual en cada lugar ${ }^{7}$. Las protestas ¿han representado un avance hacia algo parecido a la "postdemocracia"? ${ }^{8}$ En cualquiera de los casos, la desinstitucionalización implica una forma de desviación o desintegración del orden institucional ${ }^{9}$. El proceso puede llevar a la erosión de las instituciones mismas. En ocasiones, será el final de ciertos hábitos y prácticas, tras un proceso gradual, paso a paso. En el ámbito político, este carácter gradual sería quizás un inconveniente a la hora de identificar el punto de inflexión de los procesos o hacia dónde se dirige realmente el cambio: hacia dónde va la "deshabituación".

\footnotetext{
5 Della Porta, D.: Social Movements in Times of Austerity, Cambridge, Polity Press, 2015, pp. 1-25

${ }^{6}$ Hessel, S.: Indignez-vous!, Montpellier, Indigène Éditions, 2010, pp. 11-12

7 Calhoun, C.: “Occupy Wall Street in Perspective”, British Journal of Sociology, 64, 2013, pp. 26-38

${ }^{8}$ Décieux, F., Nachtwey, O. "Occupy :Protest in der Postdemokratie ", Forschungsjournal Soziale Bewegungen, 27, 2014, pp. 75-88

9 Berger, P., Luckmann, Th.: The Social Construction of Reality, p. 99
} 
La primera dificultad está en la terminología. Suele ser imprecisa, habiendo sido empleada antes, en los años setenta, para referirse a algo distinto, la demanda de estructuras y métodos alternativos para atender, dentro de la comunidad, a los pacientes con enfermedades mentales. Los objetivos eran ya entonces bastante amplios, los términos bastante difusos y controvertidos ${ }^{10}$. Las instituciones educativas fueron también objetivo de reiteradas críticas, con la pretensión de cambiar los centros organizados al modo tradicional. Por todo ello, parece necesario volver sobre el concepto y los procesos a los cuales se aplica el término.

"Desinstitucionalización" se refiere a una determinada forma de desviación y resistencia a las reglas ${ }^{11}$. No es la única modalidad de cambio. Por su carácter gradual, dificulta ver qué procesos de resistencia o desviación acabarán tocando la estructura de una institución. En el ámbito político, hay que tener presente que estos procesos pueden debilitar e incluso hacer desaparecer ciertas instituciones; así lo ha señalado el análisis institucional y, en general, la ciencia política ${ }^{12}$.

Hay que tener también en cuenta que lo institucional tiene su propia lógica, la de "lo apropiado" ${ }^{13}$. Es decir, las acciones han de "encajar" en la situación, en las rutinas y han de corresponder a expectativas realistas. Entonces, cuando llegue la pérdida de estabilidad, de legitimidad y de capacidad de integración ¿qué será "lo apropiado"? Los desafíos y movimientos, cada vez más enfrentados a los marcos de acción, pueden afectar a una parte de ese sistema, aunque es posible que dejen intactas otras reglas de funcionamiento y muchas de las prácticas "apropiadas". Los cambios acabarán llegando, pero, quizás, a aéreas distintas y en momentos distintos.

La movilización social y las protestas que irrumpieron en la política nacional e internacional en el 2011 confirman que los procesos pueden ser reversibles, al menos en parte. La cuestión es si hay algo así como una "hora cero" para las instituciones, ¿hasta qué punto es posible el vacío institucional? Tal vez se produzca la dispersión o proliferación en los procesos y en las instituciones políticas: algo parecido a un tipo de gobernanza sin gobiernos ${ }^{14}$. No es lo mismo que erosión o desaparición de sistemas y estructuras. Por ello, porque caben varias modalidades

${ }^{10}$ Bachrach, L.: Deinstitutionalization: An Analytical Review and Sociological Perspective, Rockville, National Institute of Mental Health, U.S. Department of Health, Education, and Welfare (DHEW), 1976

${ }_{11}$ Oliver, Ch.: "Strategic Responses to Institutional Processes", The Academy of Management Review, 4, 16, 1991, pp. 145-179

12 Dacin,T., Goodstein, J., Scott, R.: "Institutional Theory and Institutional Change: Introduction to the Social Research Forum", The Academy of Management Journal, 45, 2002, pp. 45-56.

13 March, J., Olsen, J.: Rediscovering Institutions, New York, The Free Press, 1989, pp. 159-172

${ }^{14}$ Hajer, M.: "Policy without Polity? Policy Analysis and the Institutional Void", Policy Sciences, 36, 2003, pp. 175-195 
de desinstitucionalización, convendría examinar más de cerca algunas de las facetas de las instituciones.

El análisis detenido de los procesos ayudará a distinguir, por ejemplo, entre aspectos normativos, cognitivos y culturales de las instituciones ${ }^{15}$. No operan de la misma forma ni tienen el mismo ritmo, pese a estar dentro de la misma institución o el mismo marco. Es probable, en fin, que haya que insistir más en las funciones que estos desempeñan - o dejan de desempeñar -, para entender qué ponen en marcha con los procesos de resistencia o de erosión. Las normas, la información y los marcos de interpretación, todo ello está en las instituciones, pero están en distintos niveles y, por ello, pueden perder vigencia o fuerza para integrar a los agentes, distintos agentes y en momentos distintos.

Hay pues, unas cuantas preguntas pendientes. Los marcos institucionales, ¿cuándo y cómo dejan de ser sistemas de integración? ¿Por qué pierden sentido o legitimidad? La desintitucionalizacion no es la respuesta sino el otro extremo de una dinámica. Es una dinámica compleja, además. Un segundo ejemplo ayudará a ver la dificultad de reemplazar sistemas completos de reglas.

\section{Reglas}

En la Ley Orgánica I/2004 se afirma que los poderes públicos no pueden ser ajenos a la violencia de género, auténtico ataque a los derechos fundamentales. Por ese motivo, ha de establecerse un sistema institucional, pensado para la tutela de las víctimas (art. 29). Es más, los poderes públicos deberán colaborar en los planes para ordenar las actuaciones en materia de violencia de género (art.32). No es tan solo un problema de índole privada, ya que pone de manifiesto la desigualdad existente entre mujeres y hombres. La Ley Orgánica 3/2007 menciona las políticas activas, a fin de garantizar la igualdad de trato y oportunidades (arts.1, 3), igualdad real y efectiva, y prevenir las conductas discriminatorias que aparecen en diferentes ámbitos.

El mandato para que se eliminen las desigualdades está dirigido a los poderes públicos (Exposición de motivos, III). En ambas normas, la del año 2004 y la del 2007, se reconoce la persistencia de la violencia y la discriminación, directa e indirecta, padecidas por las mujeres. ¿Cuál es la raíz de este grave problema? Las relaciones jerárquicas, de desigualdad, forman parte de un sistema binario, ya que las pautas sociales y culturales son, han de ser diferentes para ellas y para ellos. Queda

15 Scott, R.: Institutions and Organizations, pp. 33-62 
así definido lo que se espera de mujeres y de hombres, "lo apropiado" en cada uno de los ámbitos. Pero el predominio masculino es algo construido, no tiene base biológica: es resultado de aprendizajes y expectativas. Es una cuestión de género, no de sexo ${ }^{16}$.

La extensión y fuerte arraigo del dominio patriarcal muestran, entonces, cómo funciona un sistema consistente de reglas. En este caso, no cabe duda de que la lógica interna, lo apropiado es la desigualdad. Es un ejemplo -mal ejemplo- de procesos que generan instituciones muy estables y resistentes al cambio, en el ámbito personal y en la esfera pública. Debido a las dimensiones adquiridas por el sistema de género, la erosión o alteración de su estructura solo puede llegar con intervenciones de carácter transversal, en todas las aéreas y todos los niveles. Desde los años setenta, han ido en esta línea los argumentos defendidos por muchas de las teorías feministas: lo personal es político ${ }^{17}$.

Las instituciones son, ante todo, sistemas públicos de reglas ${ }^{18}$. Estas definen las respectivas posiciones en un sistema, con derechos y obligaciones para los agentes. Nada o muy poco alterará el funcionamiento del sistema el hecho de que las reglas sean más o menos explicitas, o que el marco sea de carácter informal o formal ${ }^{19}$. Predominan las regla ${ }^{20}$. El elemento normativo está, sin duda, en los patrones de conducta -lo apropiado-, después de que las reglas hayan establecido cómo desempeñar de la manera correcta los papeles asignados a cada agente. Indican qué obligaciones han de ser asumidas y por parte de quién. En fin, se trata de derechos y deberes. La desviación de tales reglas siempre será posible, pero tal decisión tendrá costes para quienes la tomen. Por lo tanto, se trata de sistemas públicos de obligaciones, a las que no resulta sencillo sustraerse.

La existencia de reglas de conducta es clave para la estabilidad y la legitimidad de las instituciones, también o sobre todo la de las instituciones políticas. Para contar con alguna estructura, estas necesitan un sistema de reglas, reglas formales, a modo de mecanismos de estabilidad. El sistema define las funciones, el tipo de relaciones, también el estatus de cada agente, con derechos y deberes. Las instituciones aseguran así dos de sus pilares fundamentales: el regulativo y el normativo. Pero hay más.

\footnotetext{
${ }_{16}$ Scott, J.: "Gender: A Useful Category of Historical Analysis", American Historical Review, 54, 1986, pp. 10531075

${ }_{17}$ Hanish, C.: "The Personal is Political”, Notes form the Second Year. Women's Liberation Movement, 1970, pp. $76-78$

${ }_{18}$ Rawls, J.: A Theory of Justice, Cambridge, The Belknap Press of Harvard University Press, 1999, pp. 47-52

19 Peters, G.: Institutional Theory in Political Science, London, Pinter, 1999, pp. 1-24

${ }^{20}$ Greenwood, R., Oliver, Ch., Suddaby, R., Sahlin, K.: Organizational Institutionalism, London, Sage, 2008, pp. 1-46
} 
Para funcionar, ese sistema requiere cierto grado de aceptación ${ }^{21}$, por eso ha de asentarse sobre un tercer pilar: el cognitivo ${ }^{22}$. Los sistemas públicos de reglas se apoyan, pues, en distintos elementos, incluyendo aquellos que pueden aportarles sentido. De ahí la importancia de lo cognitivo y del sistema de valores. Las reglas definen qué conducta es apropiada, en un entorno de ideas, creencias y, ante todo, de valores ${ }^{23}$. Esto quiere decir que los elementos culturales suelen ser clave en los procesos de institucionalización - también en los que van en sentido opuesto -, pues ofrecen esquemas que permitirán marcar la dirección a tomar en la vida colectiva.

Son decisivos para cualquier proceso de cambio. De ahí que el análisis institucional haya ido prestando cada vez más atención a los valores, filtros, sistemas simbólicos y, en fin, a los marcos culturales. Son básicos, aunque no sean lo único que mantiene o, por el contrario, erosiona a las instituciones. Las críticas al sistema patriarcal lo han demostrado.

\subsection{Un método}

¿Cómo Se LLEga a La Desintutucionalizacion? Existe la posibilidad de que cualquier proceso de desviación o erosión afecte de manera diferente a lo regulativo, lo normativo y lo cognitivo de una institución. Hasta ahora, las críticas sistemáticas hacia el patriarcado han insistido en los efectos adversos de un sistema binario. La distribución desigual de papeles genera, en efecto, discriminación, violencia y dominio sobre la mitad de los seres humanos. Son el resultado de procesos de habituación o institucionalización, muy extendidos y con sólidos pilares, en lo regulativo, lo normativo y lo cognitivo. Ahora bien, este sistema público de reglas -un sistema de género- está compuesto por elementos diferentes y, por ello, es o ha de ser susceptible de cambio, desviación y erosión. Solo que lo cambios pueden ser parciales o provisionales.

Con el patriarcado sucede lo mismo que en cualquier otra estructura, con un entorno situado entre la estabilidad y el cambio. Se trata, además, de un sistema de género, por tanto de distribución de papeles, aprendizajes, tradiciones y reglas de actuación. Según esto, las medidas contra la desigualdad o contra la violencia contra las mujeres han de contemplar varios niveles y fases de intervención. Las leyes son importantes, no cabe duda alguna; pero está claro que, por sí solas, no son

\footnotetext{
${ }^{21}$ Searle, J.: "What Is An Institution?", Journal of Institutional Economics, 1, 2005, pp. 1-22

22 Scott, R.: Institutions and Organizations, p. 40

23 Scott, R.: "The Adolescence of Institutional Theory”, Administrative Science Quarterly, 1987, pp. 493-511
} 
suficientes. Por eso, el método para abordar los problemas de dominación patriarcal y ponerles fin tiene un nombre: transversalidad.

La Ley Orgánica 3/2007 se refiere expresamente a la dimensión transversal de la igualdad, para intervenir en todos los ámbitos (Exposición de motivos, III). Esto significa que las actuaciones han de ser múltiples y a distintos niveles; esto vale también para abordar un problema tan grave como la violencia padecida por las mujeres. Por eso el proceso ha sido y sigue siendo tan largo y con tantas dificultades. ¿Para cuándo el fin del patriarcado? Crear un sistema de reglas alternativo, un sistema completo, requiere mucho más que la erosión de los marcos previos. Hará falta estructurar de nuevo el conjunto de las actividades, con otro sistema de valores.

Las teorías feministas contemporáneas han explorado ambas posibilidades, la crítica y la formación de un nuevo tipo de relaciones. La afirmación de que lo personal es político ha ido en esta doble dirección; no se trata solo de erosionar un sistema o una institución sino de algo de mayor alcance. Se trata de construir estructuras alternativas. Un último ejemplo mostrará cuán difícil es construir otro tipo de marcos.

\section{Otras reglas}

ENTRE El AÑo 2015 Y EL 20I7, se produjeron 21 millones de desplazamientos forzosos de población. Los datos de Naciones Unidas son concluyentes. En apenas dos años y en la Unión Europea, la llegada de importantes contingentes de refugiados y solicitantes de asilo suscitó un intenso y áspero debate social y político, sobre todo en Alemania, Hungría, Austria e Italia. En aquel momento, España solo había acogido a un 7\% de los refugiados llegados a las fronteras externas de la Unión. Las dimensiones y consecuencias de esta situación siguen siendo todavía dramáticas. Hay otro tipo de consecuencias: es un riesgo importante para la supervivencia misma del proyecto político común. ¿Por qué razón?

Europa es o debería ser un proyecto de unión política, basado en la integración y en obligaciones compartidas. Entre estas se encuentra la protección de los derechos fundamentales y de las libertades. ¿Cumple con esta obligación? Los derechos de asilo y refugio son derechos humanos y, como tales, han sido reconocidos en los acuerdos internacionales. La mayoría de los países han suscrito el Convenio de Ginebra de 1955, luego el Protocolo de 1967 sobre derechos de los refugiados. Desde el 2013 está en vigor el Reglamento de Dublín: los países europeos tienen la obligación de examinar las solicitudes 
de protección internacional. Se refiere a la protección de personas procedentes de terceros países ${ }^{24}$. Pero el cumplimiento de deberes y compromisos en esta materia es escaso.

"Constitutivas" son aquellas reglas que crean nuevas formas de comportamiento ${ }^{25}$. Los "hechos institucionales" ya existen dentro de las instituciones, con reglas propias. En muchos sentidos, la actual situación de Unión Europea muestra cuáles son las ventajas y las desventajas de las reglas constitutivas. El entramado institucional de la Unión Europea ha dado bastantes muestras de la fragilidad en su diseño político, pues las bases normativas y otros elementos constitutivos no han ido al mismo ritmo ni en la misma dirección. Las deficientes políticas migratorias y de acogida indican, lamentablemente, hasta dónde ha llegado la falta de integración en los países europeos.

La respuesta comunitaria ante la llegada de refugiados y solicitantes de asilo a las fronteras europeas es, entonces, una mala señal para la Unión. Están en riesgo tanto la capacidad de las instituciones comunitarias para garantizar derechos -los de los refugiados, pero no solo- como la legitimidad. Sobre el proyecto gravita, además, el déficit democrático o incluso la pérdida de "sustancia democrática" ${ }^{26}$. El término "europeanizacion", utilizado en ocasiones en los debates y documentación sobre el proyecto, se refiere precisamente a la singular forma de construir la política europea $^{27}$. La asimetría e incluso la asimultaneidad de las políticas de la Unión tienen, al final, consecuencias para la vida cotidiana de los europeos y para quienes proceden de otros países. La falta de integración influye, sin duda, en la percepción que los ciudadanos tienen de lo que significa "Europa" o "Bruselas".

Tal vez por eso y a lo largo de la última década, la lealtad al proyecto europeo ha ido en retroceso en varios países. El principal obstáculo para la integración política no ha estado, quizás, en la dificultad de este tipo de procesos, complejos y a ritmo variado. Ha estado en la deficiente integración y en los problemas de legitimación. Todo ello sale a relucir con ocasión de las políticas migratorias, con débil respaldo institucional y resistencias en sectores importantes de la ciudadanía. Es un hecho que el proceso de integración, al modo europeo, se encuentra en un momento especialmente crítico. ¿Ha entrado en una fase de erosión o desintitucionalización?

\footnotetext{
${ }^{24}$ Regulation (EU) No 604/2013 of the European Parliament and of the Council of 26 June 2013

25 Searle, J.: "How to Derive "Ought" From "Is", The Philosophical Review, 73, 1964, pp. 43-58

${ }^{26}$ Habermas, J.: Ach, Europa, Frankfurt, Suhrkamp, 2008, pp. 88-95; Im Sog der Tecnokratie, Frankfurt, Suhrkamp, 2013, pp. 115-124

${ }_{27}$ Radaelli, C., Featherstone, K.: "Introduction”, Featherstone, K., Radaelli, C.: The Politics of Euroepanization, Oxford, Oxford University Press, 2003, pp. 27-56
} 


\section{Conclusiones}

En el año 200 I, la Declaración sobre el futuro de la Unión europea recordaba que la legitimidad de este proyecto político deriva de los valores democráticos. De ello depende también que las instituciones mismas sean democráticas, transparentes y eficientes ${ }^{28}$. Aquellas instituciones que se apoyen en la legalidad y, además, en una justificación racional, no estarán tan sujetas al ejercicio del poder, sea este mejor o peor. Esto significa que, más allá de la formalidad jurídica, será importante contar con un tipo de justificación que esté basada en razones no contingentes, con pretensiones de validez ${ }^{29}$.

La pérdida de legitimidad puede estar en el origen de los procesos de "desinstitucionalización” de sistemas sociales y políticos. La falta de integración será también un factor de inestabilidad. Cuando esta llega, ¿se producirá el cambio de tendencia? Es probable que haya más de una respuesta a esta pregunta, ya que cualquier institución tiene una dimensión normativa -como sistema de reglas- y una dimensión cognitiva -sistemas de valores, ideas y creencias-; la principal limitación para entender los procesos de cambio está, sin embargo, en su carácter gradual. Van paso a paso, por eso a veces resulta difícil percibir el riesgo.

¿Qué puede aportar la teoría? Hacen falta conceptos adecuados y métodos para explicar la complejidad de los dos tipos de procesos, institucionalización y desintitucionalización. Hay procesos como las movilizaciones y protestas, surgidas en el 2011, también las intervenciones destinadas a poner fin al sistema patriarcal, sin olvidarse de los vaivenes de los proyectos políticos de integración, como es la Unión Europea. Las instituciones, entendidas como materialización del poder, suelen alcanzar estabilidad y solidez, pero también pueden desaparecer. Ocurrirá en el momento en que les falte lo básico: el apoyo del "poder vivo". Así llamaba H. Arendt al poder del pueblo ${ }^{30}$. Determinadas instituciones acaban petrificadas, nada más cierto. Ahora bien, importa conocer el tiempo y la forma del declive, ya que las consecuencias afectarán, afectan a todos los agentes.

\footnotetext{
${ }^{28}$ Laeken Declaration on the Future of the European Union, 2001

${ }^{29}$ Habermas, J.): Legitimationsprobleme im Spätkapitalismus, Frankfurt, Suhrkamp, 1979, pp. 133-140

${ }^{30}$ Arendt, H.: On Violence, p. 41
} 


\section{ReferenCias Bibliográficas}

Arendt, H., On Violence, Orlando, Harcourt Brace Jovanovich Publishers, 1969

Bachrach, L., Deinstitutionalization: An Analytical Review and Sociological Perspective, Rockville, National Institute of Mental Health, U.S. Department of Health, Education, and Welfare (DHEW), 1976

Berger, P., Luckmann, Th., The Social Construction of Reality, London, Penguin, 1991

Calmoun, C., "Occupy Wall Street in Perspective», British Journal of Sociology, 64, 2013, pp. 26-38

Dacin,T., Goodstein, J., Scott, R., «Institutional Theory and Institutional Change: Introduction to the Social Research Forum», The Academy of Management Journal, 45, 2002, pp. 45-56

Décieux, F., Nachtwey, O., "Occupy: Protest in der Postdemokratie », Forschungsjournal Soziale Bewegungen, 27, 2014, pp. 75-88

Della Porta, D., Social Movements in Times of Austerity, Cambridge, Polity Press, 2015

Greenwood, R., Oliver, Ch., Suddaby, R., Sahlin, K., Organizational Institutionalism, London, Sage, 2008

Habermas, J., Im Sog der Tecnokratie, Frankfurt, Suhrkamp, 2013

- Ach, Europa, Frankfurt, Suhrkamp, 2008

Hajer, M., "Policy without Polity? Policy Analysis and the Institutional Void», Policy Sciences, 36, 2003, pp. 175-195

Hanish, C., "The Personal is Political», Notes form the Second Year. Women's Liberation Movement, 1970, pp. 76-78

Hessel, S., Indignez-vous!, Montpellier, Indigène Éditions, 2010

Huntington, S., Political Order in Changing Societies, New Haven, Yale University Press, 1968

Laeken Declaration on the Future of the European Union, 2001

Ley Orgánica 1/2004, de 28 de diciembre, de Medidas de Protección Integral contra la Violencia de Género. 
Ley Orgánica 3/2007, de 22 de marzo, para la igualdad efectiva de mujeres y hombres

March, J., Olsen, J., Rediscovering Institutions, New York, The Free Press, 1989

Oliver, CH., «Strategic Responses to Institutional Processes», The Academy of Management Review, 4, 16, 1991, pp. 145-179

Parsons, T., On Institutions and Social Evolution, Chicago. The University of Chicago Press, 1982

Peters, G., Institutional Theory in Political Science, London, Pinter, 1999

Radaelli, C., Featherstone, K., "Introduction”. En: Featherstone, K., Radaelli, C.: The Politics of Europeanization, Oxford, Oxford University Press, 2003, pp. $27-56$

Rawls, J., A Theory of Justice, Cambridge, The Belknap Press of Harvard University Press, 1999

Regulation (EU) No 604/2013 of the European Parliament and of the Council of 26 June 2013

Scotт, J., "Gender: A Useful Category of Historical Analysis», American Historical Review, 54, 1986, pp. 1053-1075

Sсотт, R., Institutions and Organizations, London, Sage, 1995

— «The Adolescence of Institutional Theory», Administrative Science Quarterly, 1987, pp. 493-511

SeArLe, J., «What Is an Institution? ", Journal of Institutional Economics, 1, 2005, pp. $1-22$

— «How to Derive "Ought" From "Is" ", The Philosophical Review, 73, 1964, pp. $43-58$

DOI: http://doi.org/10.15366/bp2019.20.003

Bajo Palabra. II Época. No20. Pgs: 51-64 